\title{
Memory error in recognizing a pre-change object
}

\author{
Cheng-Ta Yang $\cdot$ Yei-Yu Yeh
}

Received: 2 April 2007 / Accepted: 24 December 2007/Published online: 30 January 2008

(C) Springer-Verlag 2008

\begin{abstract}
People often fail to detect a change between two visual scenes and retrieval failure has been suggested as a reason. We investigated the possibility that retrieval blocking underlies this failure by examining the error pattern in recognizing the pre-change object. The results of Experiment 1 showed that participants were biased toward selecting the lure that was similar to the post-change object when they failed to recognize the pre-change object. This bias was also observed in Experiment 2 when there was sufficient time to encode and consolidate the pre-change object and the bias was as strong as correct recognition in Experiment 3 when participants divided attention during encoding and comparison. The bias in memory error remained significant even when participants had the option to select an "I don't remember" response in Experiment 4. In Experiment 5, the bias was observed after participants successfully detected a change at an invalidly cued location and after they failed to detect a change at a validly cued location. These findings suggest that blocking can lead to retrieval failure in change detection when participants are aware of a change yet unable to retrieve verbatim traces and also when participants are unaware of a change and use the post-change object to retrieve the identity of the previous object at the same location.
\end{abstract}

C.-T. Yang · Y.-Y. Yeh $(\bowtie)$

Department of Psychology, National Taiwan University,

No. 1, Sec. 4, Roosevelt Rd, Taipei 106, Taiwan

e-mail: yyy@ntu.edu.tw

\section{Introduction}

The ability to store detailed visual information may be a grand illusion as people often fail to detect a large change in the visual environment (Noe \& O'Regan, 2000). The failure to detect a change, change blindness, implies that observers may not form complete representations while perceiving the visual world. This phenomenon has attracted research interest in understanding visual representation in the context of change detection (see Rensink, 2000; Simons, 2000; Simons \& Rensink, 2005 for reviews).

Researchers have suggested that change blindness arises partly because the occurrence of a post-change object overwrites the pre-change representation (Beck \& Levin, 2003; Simons, 2000). Once the pre-change representation is overwritten, its representation cannot be explicitly accessed in visual awareness. Research results supporting this view show that recognition memory of a post-change object is better than that of the pre-change object (Beck \& Levin, 2003; Mitroff, Simons, \& Levin, 2004). When an object in the pre-change array was presented alone without being replaced in the subsequent display, recognition of this object was as accurate as recognizing the post-change object that replaced the pre-change object.

Taking a different approach, Mäntylä and Sundström (2004) found a response bias in memory recall when participants failed to detect a change in a natural scene. When the pre- and post-change objects shared the same identity but different perceptual attributes (e.g., the color of a refrigerator changed), participants tended to recall the postchange attribute when asked to recall a specific attribute of the changed objects (e.g., the color of the refrigerator in the picture). After viewing a short video, participants were also biased toward recalling the post-change object when they were asked to describe the video content. Mäntylä and 
Sundström suggested that this bias arises from the fact that the activation of the post-change object is higher than that of the pre-change object because of its recency in presentation.

Despite evidence that recognition of a pre-change object is poor and participants are biased toward remembering the post-change object, a pre-change object representation does exist under change blindness according to evidence based on indirect measures such as eye movements (e.g., Hollingworth, Williams, \& Henderson, 2001; Ryan \& Cohen, 2004) and perceptual identification (Silverman \& Mack, 2006; Yeh \& Yang, 2008). After failing to detect a change in a letter matrix, the accuracy of identifying degraded pre-change letters was higher than that of identifying new letters (Silverman \& Mack, 2006), showing a repetition priming effect. Although the authors did not discuss the comparison, the priming effect for the prechange letters was similar to (Experiment 2a) or larger than the effect observed from identifying unchanged letters (Experiment 3a); the priming effect was also similar to the effect in identifying the post-change letters (Experiments $3 \mathrm{a}, \mathrm{b})$. The strength of a pre-change representation under change blindness is not less than that of an unchanged or post-change stimulus.

If the strength of a pre-change representation approaches that of an unchanged or post-change representation under change blindness, why is recognition of a pre-change object so poor (Beck \& Levin, 2003) despite being above the chance level (Mitroff et al., 2004)? What could have caused the dissociation between the indirect and direct measures of pre-change representations? Why does the conscious retrieval of a pre-change object fail? What could be the mechanism that underlies retrieval failure and the response bias in intentional recall? Prior research has not addressed the underlying mechanism that may underlie retrieval failure and recall bias when a pre-change representation under change blindness is as detailed as the representation of an unchanged or post-change object.

We considered a mechanism that underlies many memory phenomena: retrieval blocking. Blocking is a characteristic of human memory (Schacter, 2001) and has been suggested in the formal models of memory retrieval to account for empirical results (Anderson, 1983; Raaijmakers $\&$ Shiffrin, 1981). Many memory phenomena are related to blocking, such as the tip-of-the-tongue effect, the retrieval-induced forgetting effect (Anderson \& Spellman, 1995), the part-set cueing effect (Roediger, Stellon, \& Tulving, 1977), and the directed forgetting effect (Bjork, 1989; MacLeod, 1989). In the retrieval-induced forgetting effect, retrieval practice of a target exemplar of a category induces forgetting of another learned competitor exemplar. In the part-set cueing effect, the provision of a portion of the to-be-remembered items in the test phase impairs retrieval of the remaining uncued items. In the directed forgetting effect, memory of just-learned stimuli is poor if participants are instructed to forget them after the list is completed prior to learning a new list.

With its immediate accessibility due to recency, a postchange object can block participants from coming up with the correct response when attempting to access the prechange object at the same location. If this is so, we postulate that a high proportion of memory errors in recognizing the pre-change object should be biased toward selecting a critical lure that is similar to the post-change object. The purpose of this study is to examine the error pattern in recognizing the pre-change object, and to identify the context in which participants show memory errors biased toward selecting the critical lure.

Although Mäntylä and Sundström's (2004) research demonstrated a response bias toward recalling the postchange attribute under cued recall and toward recalling the post-change object after viewing a video clip, the mechanism underlying the bias remains unclear. Certain aspects in their methodology prevent a clear conclusion about the mechanism that may mediate the bias. In their first experiment in which participants were asked to report a specific attribute of the changed items, the objects were identical except for one perceptual attribute that changed between the two scenes. The bias could have arisen because participants were not specifically asked about the object's pre-change attribute. The bias in recalling the postchange object after viewing the video may have arisen from the similarity between the pre-change and postchange object in perceptual features, semantics, or both (e.g., briefcase and rucksack, book and notepad). During recall, similarity blocks the effective retrieval of the prechange object.

To examine memory error in recognizing a pre-change object, we adopted a methodology used in the literature on false memory (e.g., Roediger \& McDermott, 1995) by manipulating the recognition lures. The pre- and postchange objects were unrelated and we asked participants to recognize both the pre- and post-change object that constitute a change on each trial. There were three lures on each trial: one object that remained unchanged between two scenes, one that was conceptually similar to the prechange object (pre-change lure), and one that was conceptually similar to the post-change object (post-change lure).

The overwriting hypothesis does not specify what would occur in the error pattern. If only abstracted information is retained in a pre-change representation after overwriting (see Simons, 2000) and used in recognition, it is likely that memory error would be biased toward selecting the prechange lure when participants failed to recognize the prechange object. According to the fuzzy trace theory of false 
memory (Brainerd \& Reyna, 1996, 1998; Brainerd, Wright, Reyna, \& Payne, 2002), verbatim traces in memory representations that contain the surface details of the physical stimuli lead to accurate memory while the gist traces that represent the meaning or theme of the stimuli can lead to false memory. If this is the case, participants should falsely recognize the pre-change lure because this lure shares conceptual similarity with the pre-change object.

The blocking hypothesis compliments the retrieval failure hypothesis. According to this hypothesis, the inaccessibility of the pre-change representation results from blocking in the retrieval process as participants intentionally access a prechange object that is located in the same place as the postchange object. During the retrieval process, the gist traces of the post-change object lead to a bias in selecting the postchange lure unless participants can retrieve verbatim traces of the pre-change object. No such pattern should be observed in the recognition of the post-change object, as the postchange stimuli are immediately available. Error in postchange recognition should be biased toward selecting the unchanged object or post-change lure because of familiarity.

The prediction based on the comparison failure hypothesis (Mitroff et al., 2004; Scott-Brown, Baker, \& Orbach, 2000; Simons, Chabris, Schnur, \& Levin, 2002) depends on the assumption about the retrieval process. If a pre-change representation, be it intact or degraded, is fully retrieved but comparison fails under change blindness, the bias in memory error should be for selecting the pre-change lure because the pre-change lure is more similar to the prechange object. If the comparison failure arises from retrieval failure via blocking, more post-change lures should be falsely recognized.

We investigated memory errors in different contexts in five experiments. We adopted Beck and Levin's (2003) trial procedure in Experiment 1 using colored object images and a four-alternative forced-choice (4AFC) recognition test. We increased the stimulus duration of the pre-change array to lengthen the encoding time in Experiment 2. We investigated whether divided attention during encoding, retrieval, and comparison would increase or decrease false recognition by imposing a high concurrent load in Experiment 3. In Experiment 4, we examined whether the response bias still exists with an "I don't remember" option included in the recognition test. In Experiment 5, we investigated how focusing attention on a location during encoding may affect change detection and also the error patterns under change blindness and under correct detection.

\section{Experiment 1}

People must retrieve the pre-change representation to compare it with the post-change representation for detecting a change. We investigated the error pattern in recognizing a pre-change object and a post-change object. Participants were told that there was a change between the pre- and post-change displays on each trial and they were asked to perform two 4AFC recognition memory tests. We manipulated the distractors in the recognition test.

\section{Method}

\section{Participants}

Seven National Taiwan University undergraduate and eight graduate students volunteered in Experiment 1. The undergraduate participants received a bonus credit in an introductory psychology course for a half an hour of participation; the graduate students received our gratitude. Their ages ranged from 18 to 27. All of them had normal or corrected-to-normal vision.

\section{Apparatus}

A PC with a $3.30 \mathrm{GHz}$ Intel Pentium IV processor was used to run the experiment. A 17-in. color monitor with a vertical refresh rate of $75 \mathrm{~Hz}$. E-Prime (Schneider, Eschman, \& Zuccolotto, 2002) was used to run the experiments.

\section{Stimuli and design}

Three hundred twelve color images from the CorelDraw v5.0 (Coreldraw!, 1994) art library were used as object stimuli. Each object was converted to a 24-bit color scale and sized to a maximum of 120 pixels on both the $x$ and $y$ dimensions. Each object subtended a visual angle of $5.24^{\circ}$ (horizontal) $\times 4.29^{\circ}$ (vertical) at the viewing distance of approximately $60 \mathrm{~cm}$. Each trial used six stimuli randomly placed on a white background around an imagery circle with a diameter of $7.01^{\circ}$.

Two arrays of visual stimuli were presented in each trial. Only one object in the array changed, with the post-change object replacing the pre-change object. Forty trials were constructed with pairs of visually and conceptually dissimilar objects. Visual similarity was defined as similarity in color, size, shape, orientation, or a combination of these attributes. Conceptual similarity was defined as categorical similarity. The manipulation was verified by subjective ratings in a pilot study.

Two 4AFC recognition tasks were used to test memory of the pre-change object (pre-4AFC) and memory of the post-change object (post-4AFC). The four stimuli consisted of the pre- or post-change object (Target), an old 
unchanged object from the array (Old), a distractor sharing conceptual similarity with the pre-change object (prechange lure, PrL), and a distractor sharing conceptual similarity with the post-change object (post-change lure, PoL). Visual similarity was not manipulated because of the limited stimulus set and was random between targets and lures. Figure 1 shows an example. The pre-change object was a stapler and the post-change object was a woman's shoe. In the pre-4AFC test, the pre-change lure was a hole punch in the category of stationary and the post-change lure was a swim flipper in the category of foot wear. In the post-4AFC test, the pre-change lure was a binder clip and the post-change lure was a sports sandal.

\section{Procedure}

Six practice trials were run for participants to become familiar with the task and then 40 test trials were performed. Participants were informed that there was a change between the two arrays on each trial and that their task was to recognize the pre- and post-change objects. To suppress verbal rehearsal, participants were required to recite " 1,2 , $3,4,5$ " and " $5,4,3,2,1$ " throughout the trial until the first probe display was shown.

A fixation cross was shown for $1,000 \mathrm{~ms}$ at the beginning of each trial. After the fixation cross, the pre-change display was presented for $2,000 \mathrm{~ms}$. A blank screen was displayed during the $350 \mathrm{~ms}$ retention interval. The postchange display was presented afterward for 2,000 ms. After another interval of $350 \mathrm{~ms}$, the pre-4AFC and post$4 \mathrm{AFC}$ recognition memory tests were performed in

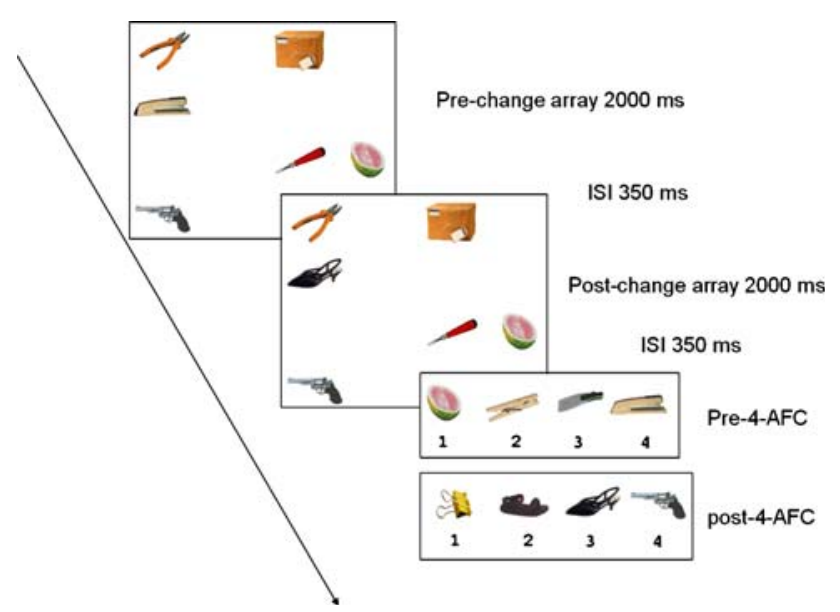

Fig. 1 A schematic diagram of the procedure used in the first three experiments. Each recognition test contains a target, an unchanged object, a pre-change lure similar to the pre-change object, and a postchange lure similar to the post-change object. Participants were asked to recognize the two objects that constitute a change between the two stimulus arrays sequence so that pre-change recognition would not be contaminated by post-change recognition. Participants were required to select the pre- and post-change objects by pressing the numeric keys $1-4$ for the test probes from left to right.

Results and discussions

\section{Data analysis}

Given the interdependency among the four choices and that the summation of response probability is 1 , we adopted the multinomial loglinear model (Agresti, 1996) in data analysis for the pre-4AFC and the post-4AFC tests separately to investigate the frequency of response types in recognition.

A multinomial loglinear model describes the log expected frequency of each response category. The loglinear model with parameters $\lambda$ can be described with the following equation:

$\log \left(\mu_{i}\right)=\lambda+\lambda_{\text {Target }}^{R}+\lambda_{\text {Old }}^{R}+\lambda_{\text {PrL }}^{R}$

The model consists of a $\log$ equation with separate parameters for each response category. As the focus was the frequency of choosing the post-change lure while recognizing the pre-change object, responses in the PoL category were chosen as the baseline category for dummy coding. Thus, the intercept describes the log expected frequency of choosing the post-change lure and the estimated parameters for the other responses describe the frequency of choosing the other stimuli.

The parameters were estimated. The goodness of fit is not reported because the model is a saturated one that contains all the parameters. The Wald test was conducted to examine whether each parameter estimate was significantly different from the frequency of the baseline response category. The estimated proportion of $\mu_{\mathrm{i}} / \mu_{\mathrm{PoL}}$ was computed to reflect the ratio between the frequency of a response type and the frequency of the $\mathrm{PoL}$ baseline response.

Table 1 presents the mean proportion of selecting the test probe in each category. Table 2 presents the significance test of each estimated parameter in contrast to the PoL response category by the Wald test.

For the pre-4AFC test, the estimated $\mu_{\mathrm{i}} / \mu_{\mathrm{PoL}}$ was 2.13 for the Target, 0.61 for the PrL category, and 0.64 for the Old category. Relative to the frequency of choosing the post-change lure, participants chose the pre-change object more often and the pre-change lures or old unchanged stimuli less often $(P<0.01)$. For the post-4AFC test, the estimated $\mu_{\mathrm{i}} / \mu_{\mathrm{PoL}}$ was 9.89 for the Target, 0.67 for the PrL category, and 1.48 for the Old category of response. The frequency of choosing the old unchanged stimulus was 
Table 1 Mean proportion and standard deviation of responses in selecting a particular test probe in Experiment 1

\begin{tabular}{lllll}
\hline & Target & Old & PrL & PoL \\
\hline Pre-4AFC & $0.49(0.12)$ & $0.14(0.05)$ & $0.14(0.05)$ & $0.23(0.07)$ \\
Post-4AFC & $0.76(0.09)$ & $0.11(0.05)$ & $0.05(0.04)$ & $0.08(0.03)$ \\
\hline
\end{tabular}

A bias in memory error was observed in recognizing the pre-change object

Target the pre-change or post-change object; Old an unchanged object in the stimulus array; $\operatorname{Pr} L$ pre-change lure that is similar to the pre-change object; $P o L$ post-change lure that is similar to the postchange object

Table 2 The results of the multinomial loglinear analysis in Experiment 1

\begin{tabular}{lllll}
\hline & \multicolumn{3}{l}{ Estimated parameters } & \\
\cline { 2 - 5 } & Intercept & $\lambda_{\text {Target }}^{R}$ & $\lambda_{\text {Old }}^{R}$ & $\lambda_{\operatorname{PrL}}^{R}$ \\
\hline Pre-4AFC & $4.92^{* *}$ & $0.76^{* *}$ & $-0.45^{* *}$ & $-0.49 * *$ \\
Post-4AFC & $3.83^{* *}$ & $2.29 * *$ & $0.39^{*}$ & -0.39 \\
\hline
\end{tabular}

The intercept describes the log expected frequency of choosing the post-change lure and the estimated parameters describe the log expected frequency of choosing the other stimuli. Asterisks indicate whether the frequency of choosing a particular stimulus is significantly different from selecting the post-change lure according to the Wald test

** $P<0.01 ; * P<0.05$

significantly $(P<0.05)$ higher than the frequency of selecting the post-change lure. The results suggest that when recognizing the post-change object, more errors occurred in selecting an old unchanged stimulus.

The proportion of correct recognition of the post-change object (0.76) was higher than that of recognizing the prechange object (0.49). When participants failed to recognize the pre-change object, they were more likely to select the post-change lure than the other two distractors. When participants failed to recognize the post-change object, they were more likely to select an unchanged object than the other distractors.

This finding rules out the possibility that the memory error in pre-change recognition is based on the overall familiarity activated by a test probe. The old unchanged stimulus was repeated twice on each trial and hence had a higher activation than both the pre-change and the postchange lures. If memory error in pre-change recognition is based on overall familiarity, participants should have chosen the old unchanged stimulus as was observed in the errors of post-change recognition. The finding is inconsistent with the prediction of the overwriting hypothesis and the version of the comparison failure hypothesis that predicts more errors in selecting the pre-change lure. Participants did not use abstracted information when recognizing the pre-change object. They used the post-change object to access the pre-change object in conscious retrieval. The error pattern is consistent with the prediction of the retrieval-blocking hypothesis.

\section{Experiment 2}

It is likely that the results of Experiment 1 arose because the pre-change object was not fully consolidated into conceptual short-term memory before the post-change array was presented. Thus, participants were biased toward selecting the lure that was conceptually similar to the postchange object when they failed to recognize the pre-change object. We increased the duration of the pre-change array to $3,600 \mathrm{~ms}$ in this experiment. With six objects in the array, each object can be processed for $600 \mathrm{~ms}$ if participants serially encode each one. As it takes about $400 \mathrm{~ms}$ to access the semantics of a complex picture in conceptual short-term memory (Potter, 1976), each pre-change object should be processed to the semantic level in the current context.

\section{Method}

\section{Participants}

Thirty undergraduate students volunteered for Experiment 2. Each participant received a bonus credit in an introductory psychology course for a half hour of participation. Their ages ranged from 18 to 22. All of them had normal or corrected-to-normal vision.

\section{Design, stimuli, and procedure}

The design and stimuli were the same as those used in Experiment 1 except that the duration of the pre-change array was increased from 2,000 to 3,600 ms. Participants were asked to suppress verbal rehearsal while viewing the pre- and post-change displays.

\section{Results and discussion}

Table 3 presents the mean proportion of responses selecting the test probe in each category. Table 4 presents the significance test using the Wald test.

For the pre-4AFC test, the estimated $\mu_{\mathrm{i}} / \mu_{\mathrm{PoL}}$ was 3.15 for the Target, 0.58 for the PrL category, and 0.64 for the Old category of response. Memory error occurred more frequently in the PoL category than in the PrL and Old categories $(P<0.01)$. For the post-4AFC test, the 
Table 3 Mean proportion and standard deviation of responses in selecting a particular test probe in Experiment 2

\begin{tabular}{lllll}
\hline & Target & Old & PrL & PoL \\
\hline Pre-4AFC & $0.59(0.12)$ & $0.11(0.04)$ & $0.11(0.04)$ & $0.19(0.12)$ \\
Post-4AFC & $0.80(0.08)$ & $0.08(0.05)$ & $0.05(0.03)$ & $0.07(0.04)$ \\
\hline
\end{tabular}

A bias in memory error was observed in recognizing the pre-change object

Target the pre-change or post-change object, Old an unchanged object in the stimulus array; $\operatorname{PrL}$ pre-change lure; $P o L$ post-change lure

Table 4 The results of the multinomial loglinear analysis in Experiment 2

\begin{tabular}{lllll}
\hline & \multicolumn{4}{l}{ Estimated parameters } \\
\cline { 2 - 5 } & Intercept & $\lambda_{\text {Target }}^{R}$ & $\lambda_{\mathrm{Old}}^{R}$ & $\lambda_{\mathrm{PrL}}^{R}$ \\
\hline Pre-4AFC & $5.38^{* *}$ & $1.15^{* *}$ & $-0.45^{* *}$ & $-0.54^{* *}$ \\
Post-4AFC & $4.32^{* *}$ & $2.51^{* *}$ & $0.36^{* *}$ & $-0.35 \dagger$ \\
\hline
\end{tabular}

The intercept describes the log expected frequency of choosing the post-change lure and the estimated parameters describe the log expected frequency of choosing the other stimuli. Asterisks indicate whether the frequency of choosing a particular stimulus is significantly different from selecting the post-change lure according to the Wald test

** $P<0.01 ; \dagger P=0.053$

estimated $\mu_{\mathrm{i}} / \mu_{\mathrm{PoL}}$ was 12.32 for the Target, 0.71 for the PrL category, and 1.43 for the Old category. The frequency of selecting the old unchanged stimulus was significantly higher $(P<0.01)$ than the frequency of choosing the postchange lure. When they erred in the recognition of the postchange object, participants were biased towed selecting the old unchanged stimulus.

Results were similar to those of Experiment 1. Although lengthening the stimulus duration increased the proportion of correct recognitions of the pre-change object from 0.49 in Experiment 1 to 0.59 in Experiment 2, pre-change recognition was still worse than recognition of the postchange object (0.80). Participants were biased toward selecting the post-change lure when they erred in recognizing the pre-change object. Participants chose an old unchanged stimulus when they made a mistake in recognizing the post-change object.

\section{Experiment 3}

The results of the first two experiments showed a bias in the error pattern when participants failed to recognize the pre-change object even when they had sufficient time to encode each pre-change stimulus serially. The results may have arisen because the participants divided their attention among all the stimuli in the pre-change display and focused only on the changed object when encoding the post-change display. The bias in memory error arose because of the elaborative encoding of the post-change object.

In this experiment, we examined what would happen if elaborative encoding of the post-change object is reduced under divided attention. It is possible that under divided attention participants would not be able to elaborately encode the post-change object and so would not be able to relate the pre- and post-change objects in comparison. As a result, the bias in the error pattern would be reduced. Alternatively, it is likely that both the pre- and post-change objects will be encoded to a lesser degree under divided attention. As a result, the likelihood of using the postchange object to retrieve the pre-change object will increase and the bias in the error pattern will become stronger.

\section{Method}

\section{Participants}

Eleven undergraduate students volunteered in Experiment 3. Each participant received a bonus credit in an introductory psychology course for a half an hour of participation. Their ages ranged from 18 to 22 . All of them had normal or corrected-to-normal vision.

\section{Design, stimuli, and procedure}

All aspects were identical to those used in Experiment 2 except that an additional task was performed until the memory probes occurred. In the beginning of each trial, a two-digit number was presented in the center of the screen. Participants were asked to count backward by three while viewing the stimuli until the recognition memory tests. The concurrent load not only suppresses verbal coding of the objects but also divides participants' attention while encoding and comparing the pre- and post-change stimuli.

\section{Results and discussion}

Table 5 presents the mean proportion of responses selecting the test probe in each category. Table 6 presents the significance test using the Wald test.

For the pre-4AFC test, the estimated $\mu_{\mathrm{i}} / \mu_{\mathrm{PoL}}$ was 0.97 for the Target, 0.38 for the PrL category, and 0.40 for the Old category. There was no significant difference in the frequency of selecting the pre-change object and the postchange lure $(P>0.1)$. The frequency of memory error for the post-change lure was significantly higher $(P<0.01)$ 
Table 5 Mean proportion and standard deviation of responses in selecting a particular test probe in Experiment 3

\begin{tabular}{lllll}
\hline & Target & Old & PrL & PoL \\
\hline Pre-4AFC & $0.33(0.11)$ & $0.15(0.08)$ & $0.15(0.04)$ & $0.37(0.15)$ \\
Post-4AFC & $0.63(0.13)$ & $0.15(0.06)$ & $0.11(0.07)$ & $0.11(0.05)$ \\
\hline
\end{tabular}

The frequency of selecting the post-change lure in pre-change recognition was as high as the hit rate of correct recognition

Target the pre-change or post-change object; Old an unchanged object in the stimulus array; $\operatorname{PrL}$ pre-change lure; $P o L$ post-change lure

Table 6 The results of the multinomial loglinear analysis in Experiment 3

\begin{tabular}{lllll}
\hline & \multicolumn{4}{l}{ Estimated parameters } \\
\cline { 2 - 5 } & Intercept & $\lambda_{\text {Target }}^{R}$ & $\lambda_{\mathrm{Old}}^{R}$ & $\lambda_{\mathrm{PrL}}^{R}$ \\
\hline Pre-4AFC & $4.98 * *$ & -0.03 & $-0.92^{* *}$ & $-0.98^{* *}$ \\
Post-4AFC & $3.71^{* *}$ & $1.85^{* *}$ & $0.35 \dagger$ & -0.05 \\
\hline
\end{tabular}

The intercept describes the log expected frequency of choosing the post-change lure and the estimated parameters describe the log expected frequency of choosing the other stimuli. Asterisks indicate whether the frequency of choosing a particular stimulus is significantly different from selecting the post-change lure according to the Wald test

** $P<0.01 ; \dagger P=0.056$

than the frequency of error based on the old unchanged object and the pre-change lure. For the post-4AFC test, the estimated $\mu_{\mathrm{i}} / \mu_{\mathrm{PoL}}$ was 6.39 for the Target, 0.95 for the PrL category, and 1.41 for the Old category. Participants were biased toward selecting an old unchanged stimulus $(P=0.056)$ in comparison with the frequency of choosing the post-change lure when they erred in recognizing the post-change object.

The concurrent load lowered the hit rate in the recognition of both the pre- and post-change objects. With the same encoding duration for the pre-change array as in Experiment 2 , the proportion of correct recognition of the pre-change object decreased from 0.59 to 0.33 . With the same encoding duration for the post-change array as in Experiments 1 and 2, the proportion of correct recognition of the post-change object deteriorated from 0.76 (Experiment 1) and 0.80 (Experiment 2) to 0.63 in this experiment. Decreasing attention resources to encoding increased memory errors.

Decreasing the degree of elaborative encoding of postchange stimuli did not reduce the bias in memory error. Instead, memory error based on the post-change lure was as high as the hit rate in the recognition of the pre-change object. The frequency of false recognition of the postchange lure increased from 0.19 in Experiment 2 to 0.37 in this experiment given the same encoding duration for the pre-change array. Using a concurrent task to divide attention in encoding, Pérez-Mata, Read, and Diges (2002) also found that the frequency of false recall of a lure that represented the theme of each study list doubled under divided attention.

When both the pre- and post-change objects were encoded under divided attention, both representations contained fewer verbatim traces. The increase in memory error arose because participants relied more on the gist traces of the post-change object to retrieve the pre-change representation that did not contain much detail in the verbatim traces. Participants chose an old unchanged object more often when they erred in the recognition of the postchange object.

\section{Experiment 4}

Results from previous three experiments suggest that there is a bias toward selecting a post-change lure when participants fail to recognize the pre-change object. The error rate for the post-change lure increased when the participants were required to divide their attention during encoding, retrieval, and comparison. However, it is possible that the bias is an artifact resulting from limiting the choices in the recognition test. Thus, we added an "I don't remember" option to the recognition test so that participants could indicate when they realize they do not remember the exact answer. We examined the bias in memory error using a $5 \mathrm{AFC}$ recognition test.

\section{Method}

\section{Participants}

Twenty-two undergraduate students volunteered for Experiment 4. Each participant received NT\$50 (US\$1.70) for a half hour of participation. Their ages ranged from 20 to 24. All of them had normal or corrected-to-normal vision.

\section{Design, stimuli, and procedure}

The design and stimuli were similar to those used in Experiment 1 except for 2 modifications. First, 2 fivealternative-forced-choice (5AFC) recognition memory tests were used by adding an "I don't remember" option. Second, 6 Mondrian patterns were presented $350 \mathrm{~ms}$ after the post-change display for a duration of $2,000 \mathrm{~ms}$ so that both the pre- and post-change arrays were followed by another visual display. Following the mask display, the pre- and post-5AFC tests were tested in sequence. 
Results and discussion

Table 7 presents the mean proportion of responses selecting the test probe in each category. Table 8 presents the significance test using the Wald test.

Similar to Experiments 1-3, we used a loglinear model to analyze the frequency of each category, and the PoL was selected as the baseline category. For the pre-5AFC test, the estimated $\mu_{\mathrm{i}} / \mu_{\mathrm{PoL}}$ was 2.83 for the Target, 0.79 for the PrL category, 0.73 for the Old category, and 0.55 for the "I don't remember" option. The results showed that participants were still biased toward selecting the post-change lure even with an added "I don't remember" option in the recognition of the pre-change object. The frequency of selecting the post-change lure was significantly higher than that of selecting the old unchanged stimulus $(P<0.05)$ and the "I don't remember" option $(P<0.01)$. The difference in the frequency of selecting the post-change lure and the pre-change lure approached significance $(P=0.06)$. For the post-5AFC test, the estimated $\mu_{\mathrm{i}} / \mu_{\mathrm{PoL}}$ was 16.93 for the Target, 0.60 for the PrL category, 1.55 for the Old category, and 0.88 for the "I don't remember" option. Participants were biased toward selecting an old unchanged stimulus $(P<0.05)$ when they erred in the recognition of the post-change object.

The results remained similar to those found in the previous three experiments even when a mask display was presented after the post-change array and the "I don't remember" option was added to the recognition test. The hit rate for the pre-change object $(0.48)$ was similar to that in Experiment $1(0.49)$ using the same duration for the prechange display. Although the error rate for the post-change lure (0.17) was lower than that in Experiment 1 (0.23), this error rate was still higher than that for the other choices. Participants chose an old unchanged object when they erred in recognizing the post-change object.

\section{Experiment 5}

The results of the four experiments showed a bias in the error pattern when participants failed to recognize the prechange object. The bias was strong when participants had to divide their attention with another task during encoding,
Table 8 The results of the multinomial loglinear analysis in Experiment 4

\begin{tabular}{llllll}
\hline \multicolumn{5}{l}{ Estimated parameters } \\
\cline { 2 - 6 } & Intercept & $\lambda_{\text {Target }}^{R}$ & $\lambda_{\mathrm{Old}}^{R}$ & $\lambda_{\mathrm{PrL}}^{R}$ & $\lambda_{\text {Forget }}^{R}$ \\
\hline Pre-5AFC & $5.00^{* *}$ & $-0.60 * *$ & $-0.31 *$ & $-0.23 \dagger$ & $1.04 * *$ \\
Post-5AFC & $3.74 * *$ & $2.83 * *$ & $0.44 *$ & $-0.52 *$ & -0.13 \\
\hline
\end{tabular}

The intercept describes the log expected frequency of choosing the post-change lure and the estimated parameters describe the log expected frequency of choosing the other stimuli. Asterisks indicate whether the frequency of choosing a particular stimulus is significantly different from selecting the post-change lure according to the Wald test

** $P<0.01 ; * P<0.05 ; \dagger P=0.058$

retrieval, and comparison. However, the bias in memory error may have arisen because the post-change object was always the one in focal attention prior to detecting the change even under divided attention. It was the encoding of the post-change object that reduced the accessibility of the pre-change representation.

We examined this possibility by presenting an uninformative cue prior to the pre-change display. As a cue onset attracts exogenous attention (Hopfinger \& Mangun, 1998; Yantis \& Jonides, 1984), it enables the cued stimulus to be transferred into visual working memory despite the unpredictability of the cue (Schmidt, Vogel, Woodman, \& Luck, 2002). Following Schmidt et al.'s (2002) cuing procedure, a cue box was presented before the pre-change display. The cue box was uninformative with a $50 \%$ probability of indicating the location of the changed object in the change trials. We investigated the error pattern as a function of cue validity under change blindness and correct detection.

\section{Method}

\section{Participants}

Twenty-nine undergraduate students volunteered in Experiment 5. Each participant received NT\$120 (US\$4) for an hour of participation. Their ages ranged from 18 to 24. All of them had normal or corrected-to-normal vision.

Table 7 Mean proportion and standard deviation of responses in selecting a particular test probe in Experiment 4

\begin{tabular}{llllll}
\hline & Target & Old & PrL & PoL & Forget \\
\hline Pre-5AFC & $0.48(0.10)$ & $0.12(0.05)$ & $0.13(0.06)$ & $0.17(0.10)$ & $0.09(0.10)$ \\
Post-5AFC & $0.81(0.08)$ & $0.07(0.05)$ & $0.03(0.03)$ & $0.05(0.04)$ & $0.04(0.04)$ \\
\hline
\end{tabular}

A bias in memory error was observed in recognizing the pre-change object

Target the pre-change or post-change object; Old an unchanged object in the stimulus array; PrL pre-change lure; PoL post-change lure; Forget "I don't remember" 


\section{Design, stimuli, and procedure}

There were three major modifications in this experiment. First, 160 trials were constructed with half as no-change trials and half as the change trials. In the no-change trials, the six objects in the pre-change displays were identical to those in the post-change displays. To construct another 40 change trials, another set of 40 pairs of unrelated pre- and postchange objects were selected by three independent raters. Second, a black box was presented as an onset cue before the pre-change displays for $100 \mathrm{~ms}$. The cue was $50 \%$ valid in the change trials. In the no-change trials, the cued position was randomly selected from the six positions. In the change trials, the cue indicated the change location in half of the trials (valid-cue condition) and indicated one of the five nonchange locations in another half (invalid-cue condition). Third, participants were asked to perform a change detection task prior to the recognition memory tests. Accuracy rather than speed was emphasized for change detection. The preand post-5AFC recognition memory tests were performed only in the change trials regardless of the detection decision.

Results and discussion

\section{Change detection}

The proportion of correct detection was analyzed with a one-way repeated measures analysis of variance (ANOVA) to examine the cue effect on detection performance. The main effect of cue validity was significant $\left[F(1,28)=7.30, P<0.05, \eta^{2}=0.21\right]$, showing that detection performance was better in the valid-cue condition (0.91) than that in the invalid-cue condition (0.88). A valid cue aids participants in detecting a change.

\section{Recognition performance}

Table 9 shows recognition performance as a function of cue validity and detection accuracy. The recognition memory hit rates were analyzed with a 2 (detection accuracy) $\times 2$ (cue validity) repeated measures ANOVA for the pre- and post-change objects. For the pre-change memory, the main effect of the detection accuracy was significant $\left[F(1,28)=266.21, \quad P<0.01, \quad \eta^{2}=0.90\right]$, showing that the recognition memory was better under correct detection (0.59) than that under detection failure (0.16). The main effect of the cue validity was significant $\left[F(1,28)=5.12, P<0.05, \eta^{2}=0.16\right]$, showing that the hit rate was higher when the cue was valid $(0.39)$ than when the cue was invalid (0.34). The interaction between detection accuracy and cue validity was also significant $\left[F(1,28)=8.20, P<0.01, \eta^{2}=0.23\right]$. The validity effect was significant only when the change was detected. In contrast, there was no difference between the valid-cue and invalid-cue conditions when the change was not detected. For the post-change memory, the main effect of detection

Table 9 Mean proportion and standard deviation of responses in selecting a particular test probe in Experiment 5

\begin{tabular}{|c|c|c|c|c|c|}
\hline & Target & Old & PrL & PoL & Forget \\
\hline \multicolumn{6}{|c|}{ Detection failure } \\
\hline \multicolumn{6}{|l|}{ Invalid cue } \\
\hline Pre-5AFC & $0.14(0.16)$ & $0.18(0.22)$ & $0.05(0.14)$ & $0.17(0.22)$ & $0.46(0.29)$ \\
\hline Post-5AFC & $0.43(0.31)$ & $0.13(0.17)$ & $0.04(0.09)$ & $0.12(0.22)$ & $0.29(0.32)$ \\
\hline \multicolumn{6}{|l|}{ Valid cue } \\
\hline Pre-5AFC & $0.12(0.18)$ & $0.15(0.23)$ & $0.15(0.19)$ & $0.28(0.30)$ & $0.27(0.28)$ \\
\hline Post-5AFC & $0.37(0.32)$ & $0.09(0.15)$ & $0.11(0.17)$ & $0.11(0.23)$ & $0.27(0.33)$ \\
\hline \multicolumn{6}{|c|}{ Correct detection } \\
\hline \multicolumn{6}{|l|}{ Invalid cue } \\
\hline Pre-5AFC & $0.53(0.14)$ & $0.10(0.05)$ & $0.10(0.06)$ & $0.16(0.07)$ & $0.11(0.14)$ \\
\hline Post-5AFC & $0.88(0.08)$ & $0.03(0.03)$ & $0.02(0.02)$ & $0.04(0.04)$ & $0.02(0.03)$ \\
\hline \multicolumn{6}{|l|}{ Valid cue } \\
\hline Pre-5AFC & $0.65(0.10)$ & $0.09(0.06)$ & $0.08(0.05)$ & $0.10(0.06)$ & $0.06(0.08)$ \\
\hline Post-5AFC & $0.87(0.07)$ & $0.03(0.03)$ & $0.03(0.03)$ & $0.07(0.04)$ & $0.01(0.02)$ \\
\hline
\end{tabular}

Participants predominantly chose the "I don't remember" option under detection failure, but false recognition of the post-change lure increased in the valid-cue condition under detection failure. False recognition in pre-change recognition was also observed in the invalid-cue condition under correct detection

Target the pre-change or post-change object; Old an unchanged object in the stimulus array; PrL pre-change lure; PoL post-change lure; Forget "I don't remember" 
Table 10 The results of the multinomial loglinear analysis in Experiment 5

\begin{tabular}{|c|c|c|c|c|c|}
\hline & \multicolumn{5}{|c|}{ Estimated parameters } \\
\hline & Intercept & $\lambda_{\text {Target }}^{R}$ & $\lambda_{\text {Old }}^{R}$ & $\lambda_{\mathrm{PrL}}^{R}$ & $\lambda_{\text {Forget }}^{R}$ \\
\hline \multicolumn{6}{|c|}{ Detection failure } \\
\hline \multicolumn{6}{|l|}{ Invalid cue } \\
\hline Pre-5AFC & $3.22 * *$ & -0.17 & -0.17 & $-0.92 *$ & $0.86^{* *}$ \\
\hline Post-5AFC & $2.71 * *$ & $1.35 * *$ & 0.38 & -0.76 & $0.81 * *$ \\
\hline \multicolumn{6}{|l|}{ Valid cue } \\
\hline Pre-5AFC & $3.37 * *$ & $-0.88^{*}$ & $-0.72 *$ & $-0.73^{*}$ & 0.13 \\
\hline Post-5AFC & $2.30 * *$ & $1.41 * *$ & -0.11 & 0.26 & $1.06 * *$ \\
\hline \multicolumn{6}{|c|}{ Correct detection } \\
\hline \multicolumn{6}{|l|}{ Invalid cue } \\
\hline Pre-5AFC & $5.08 * *$ & $1.22 * *$ & $-0.41 * *$ & $-0.50 * *$ & $-0.38 * *$ \\
\hline Post-5AFC & $3.83 * *$ & $2.98 * *$ & -0.33 & $-0.74 * *$ & $-0.94 * *$ \\
\hline \multicolumn{6}{|l|}{ Valid cue } \\
\hline Pre-5AFC & $4.69 * *$ & $1.89 * *$ & -0.10 & -0.24 & $-0.47 * *$ \\
\hline Post-5AFC & $4.30 * *$ & $2.54 * *$ & $-0.84 * *$ & $-0.97 * *$ & $-1.82 * *$ \\
\hline
\end{tabular}

The intercept describes the log expected frequency of choosing the post-change lure and the estimated parameters describe the log expected frequency of choosing the other stimuli. Asterisks indicate whether the frequency of choosing a particular stimulus is significantly different from selecting the post-change lure according to the Wald test

** $P<0.01 ; * P<0.05$

accuracy was significant $[F(1,28)=111.63, P<0.01$, $\left.\eta^{2}=0.80\right]$, showing that recognition memory was better under correct detection (0.88) than under detection failure (0.40). No other effects reached a significant level $(P>0.1)$. Memory of both the pre- and post-change objects was better under correct detection than under detection failure. A valid cue improved pre-change recognition only when participants could accurately detect the change. Cue validity did not affect the hit rates in recognizing the post-change object.

The error pattern in recognition was analyzed by loglinear models separately according to the condition defined by the test (pre-/post-5AFC), cue validity (invalid/valid cue), and detection accuracy (detection failure/correct detection). Table 10 presents the significance test using the Wald test.

\section{Memory errors with an invalid cue under detection failure}

For the pre-5AFC test, the estimated $\mu_{\mathrm{i}} / \mu_{\mathrm{PoL}}$ was 0.84 for the Target, 0.40 for the PrL category, 0.84 for the Old category, and 2.36 for the "I don't remember" option. When participants could not recognize the pre-change object, they chose the "I don't remember" option with a higher frequency than they selected the post-change lure
$(P<0.01)$. The frequency of selecting the post-change lure was higher than that of selecting the pre-change lure $(P<0.05)$, and not significantly different from that of choosing the pre-change object or of choosing an old unchanged stimulus $(P>0.1)$. There was no bias for choosing the post-change lure in pre-change recognition. For the post-5AFC test, the estimated $\mu_{\mathrm{i}} / \mu_{\mathrm{PoL}}$ was 3.87 for the Target, 0.47 for the PrL category, 1.47 for the Old category, and 2.27 for the "I don't remember" option. Participants again selected the "I don't remember" option when failing to recognize the post-change object and the frequency was significantly higher $(P<0.01)$ than that of selecting the post-change lure.

\section{Memory errors with a valid cue under detection failure}

For the pre-5AFC test, the estimated $\mu_{\mathrm{i}} / \mu_{\mathrm{PoL}}$ was 0.41 for the Target, 0.48 for the PrL category, 0.48 for the Old category, and 1.14 for the "I don't remember" option. When participants failed to recognize a pre-change object, the frequency of selecting the post-change lure was not significantly different $(P>0.1)$ from choosing the "I don't remember" option. The frequency of selecting the postchange lure was significantly higher than that in choosing the pre-change object $(P<0.05)$, an old unchanged stimulus $(P<0.05)$, or the pre-change lure $(P<0.05)$. The cue increased the frequency of choosing the post-change lure in memory error. For the post-5AFC test, the estimated $\mu_{\mathrm{i}} / \mu_{\mathrm{PoL}}$ was 4.10 for the Target, 1.30 for the PrL category, 0.90 for the Old category, and 2.90 for the "I don't remember" option. When participants erred in recognizing the post-change object, they tended to choose the "I don't remember" option $(P<0.01)$.

\section{Memory errors with an invalid cue under correct detection}

For the pre-5AFC test, the estimated $\mu_{\mathrm{i}} / \mu_{\mathrm{PoL}}$ was 3.40 for the Target, 0.61 for the PrL category, 0.66 for the Old category, and 0.68 for the "I don't remember" option. When participants failed to recognize a pre-change object, participants were more likely to choose the post-change lure. The frequency in choosing this response was significantly higher $(P<0.01)$ than that in selecting an old unchanged stimulus, the pre-change lure, and the "I don't remember" category. False recognition of the post-change lure occurred. For the post-5AFC test, the estimated $\mu_{\mathrm{i}} / \mu_{\mathrm{PoL}}$ was 19.67 for the Target, 0.48 for the PrL category, 0.72 for the Old category, and 0.39 for the "I don't remember" option. When participants erred in recognizing the postchange object, they tended to select either an old unchanged object or the post-change lure, as there was no 
significant difference $(P>0.1)$ in the frequency of these two categories of response.

\section{Memory errors with a valid cue under correct detection}

For the pre-5AFC test, the estimated $\mu_{\mathrm{i}} / \mu_{\mathrm{PoL}}$ was 6.65 for the Target, 0.79 for the PrL category, 0.91 for the Old category, and 0.62 for the "I don't remember" option. The frequency in selecting the post-change lure was significantly higher than that in choosing the "I don't remember" option $(P<0.01)$, but was not significantly different from that in choosing the old stimulus or the pre-change lure $(P>0.1)$. There was no bias in error toward the post-change lure when participants erred in recognizing the pre-change object. For the post-5AFC test, the estimated $\mu_{\mathrm{i}} / \mu_{\mathrm{PoL}}$ was 12.72 for the Target, 0.38 for the PrL, 0.43 for the Old category, and 0.16 for the "I don't remember" option. When participants erred in recognizing the post-change object, they were biased toward the post-change lure $(P<0.01)$.

The performance in change detection was better when the cue was valid than when the cue was invalid. Despite the fact that the cue was uninformative, participants used the cue to detect whether a change had occurred. Given that a valid cue attracts exogenous attention and transfers the perceptual representation of the pre-change object into working memory, participants could successfully compare the post-change object with the pre-change representation. A change was more likely to be detected in the valid-cue condition.

Change detection could still fail in the valid-cue condition. It appears that the participants used the object at the cued location to retrieve the pre-change object in recognition after failing to detect a change. In the invalid-cue condition, this strategy failed to retrieve any elements that resembled the ones in the test probes, as the object at the cued location was unchanged. Participants hence adopted a recognition strategy such as "If I cannot detect the change and cannot remember any feature elements shown in the test probes, it must have been that I do not have any exact memory of the two objects that constitute the change". Thus, participants predominantly chose the "I don't remember" option when they failed to recognize either the pre-change or the post-change object under change blindness. Situational and metacognitive knowledge about how memory should work can be used to reduce false memory (Libby \& Neisser, 2001).

In the valid-cue condition under change blindness, the cued object was the post-change object. The use of the post-change object as the retrieval cue reduced the "I don't remember" response and the frequency of selecting the post-change lure increased. Both responses were chosen at a higher rate than the pre-change object. An interesting observation is that participants did not use the cue in recognizing the post-change object, as the error pattern in post-change recognition remained similar between the invalid-cue and valid-cue conditions.

A location-based retrieval strategy can also lead to false recognition under correct detection. As the cue attracted attention, participants focused their attention on the object at the cued location in both displays. In the invalid-cue condition when participants realized that the object had not changed in the second display, they had to re-distribute their attention until identifying the change for correct detection. The post-change object was in focal attention whereas the pre-change object may not have been. False recognition arose if participants used the focused postchange object to retrieve the pre-change object.

Given that the error pattern varied as a function of detection accuracy and cue validity, it is unlikely that the encoding of a post-change object is the main reason for observing the bias in memory error of pre-change recognition. According to the overwriting hypothesis, the encoding of the post-change object disrupts the pre-change representation that can support correct change detection but not accurate memory. If the volatile pre-change representation is used under both correct detection and change blindness, recognition performance should be independent of cue validity and detection accuracy. If the encoding of the post-change object blocks the retrieval of the prechange object only when the pre-change object is not in focal attention, the error pattern should be independent of detection accuracy when the cue is invalid. And, the frequency of selecting the post-change lure should not increase in the valid-cue condition under detection failure.

\section{General discussion}

The objective of the current study was to investigate the error pattern in recognizing the pre-change and postchange objects in the context of change detection. We emulated the first attempt at retrieving a pre-change representation for change detection in Experiments 1-4. Participants were asked to recognize the two objects that constituted a change. The results showed a bias in the error pattern when participants failed to recognize the prechange object. Participants falsely recognized as the prechange object a post-change lure that shared conceptual similarity with the post-change object. No such bias existed in recognizing the post-change object. Memory error was mostly with the old unchanged object when failing to recognize the post-change object. The bias in the error pattern of pre-change recognition was observed when there was sufficient time to consolidate each object in Experiment 2 and was as high as the hit rate when participants 
had to divide their attention between two tasks in Experiment 3. Even when the "I don't remember" option was provided in Experiment 4, false recognition was still observed in pre-change recognition.

When an uninformative cue was presented before the pre-change display in Experiment 5, detection performance was better if the change occurred at the cued location than if the change occurred at another location. After correct detection, false recognition was observed in the invalid-cue condition, but not in the valid-cue condition. After detection failure, false recognition in the valid-cue condition was higher than the hit rate and was similar to the "I don't remember" option. No false recognition was observed in the invalid-cue condition under change blindness.

\section{Attention and change blindness}

Attention indeed plays an important role in change detection (Rensink, 2000). The results of both Experiments 3 and 5 support this critical role of attention in object memory and change detection. Under divided attention, elaborative encoding is unlikely and both the pre- and postchange objects contain less visual detail in the verbatim traces. Divided attention decreased the hit rates for recognizing both the pre- and post-change objects. And, the bias in selecting the post-change lure was as strong as the hit rate for recognizing the pre-change object. When an uninformative cue captures attention and enables the cued stimulus to be transferred into visual working memory with a higher priority (Schmidt et al., 2002), the cued representation contains more detailed information in its verbatim traces than the uncued ones. When a change does occur at the cued location, participants are more accurate at detecting the change. And, they are better at recognizing the cued pre-change object after correct detection.

However, focal attention to an object is not sufficient to guarantee correct change detection. After the cue attracts attention and prioritizes the cued object to visual working memory, participants may divert attention to other uncued objects in either the pre-change display or the post-change display. Without attention to both objects for comparison, change detection fails. Even when both the pre- and postchange objects are attended to, change detection can still fail because of a failure in memory retrieval (Hollingworth, 2003) or in comparison (Mitroff et al., 2004; Scott-Brown et al., 2000).

\section{Memory error}

Human memory can be distorted (Schacter, 2001). Memory error is as likely as true memory with the use of the Deese-
Roediger-McDermott (DRM) paradigm (Roediger \& McDermott, 1995). In the DRM paradigm, participants learn a series of words (e.g., kid, adult, and adolescent) relating to an unseen lure (e.g., CHILD). In the test phase, participants are asked either to recall or recognize items learned in the study phase. Although participants never see the critical lure (CHILD) in the study phase, they inaccurately recall or recognize this critical lure as a studied stimulus. A picture paradigm has also been developed to demonstrate false recognition of an object in a picture with a thematic scene (Miller \& Gazzaniga, 1998). Exemplars that did not occur in the original pictures were falsely recognized at a rate equivalent to the true recognition of objects that did occur in the scenes.

The results of the current study show that false recognition can arise in the context of change detection even when the pre- and post-change objects are unrelated. When they failed to recognize a pre-change object, participants were biased toward selecting a post-change lure that shares conceptual similarity with the post-change object. This false recognition manifested even when participants had the option of choosing "I don't remember" as the answer in Experiment 4, and even after participants could correctly detect a change at an invalidly cued location in Experiment 5.

False recognition of a pre-change object appears to be mediated by a location-based retrieval strategy. The location was specified by either the cue or the post-change object. False recognition was observed unless participants failed to detect a change occurring at an invalidly cued location or unless participants correctly detected a change that occurred at the cued location. In the former context, the use of the object at the cued location in retrieval does not reactivate any elements in the test probes and the knowledge that they failed to detect a change would indicate to the participants that they had no awareness of the two objects that constituted the change. Belief about how memory should work reduces false memory (Libby \& Neisser, 2001). In the latter context, elaborative encoding of the pre-change object at the cued location leaves greater detail in the verbatim traces that are successfully retrieved for correct detection. Verbatim traces lead to accurate memory (Brainerd \& Reyna, 1996, 1998). Except in these two contexts, the use of a post-change object to identify the previous object at the same location can lead to false recognition.

The observation of false recognition of a pre-change object is quite interesting as the interval from encoding the pre-change display to the test of a pre-change object was quite brief, ranging from $4.7 \mathrm{~s}$ (Experiment 1) to $6.3 \mathrm{~s}$ (Experiments 2 and 3 ) to an interval of $7.05 \mathrm{~s}$ (Experiments 4 and 5). In contrast, the interval was much longer in the studies that investigated false memory. The parallel finding 
of memory errors is in line with the suggestion of the continuity of the evaluative process recruited in working memory and long-term memory via common neural mechanisms (Koch et al., 2006; Mitchell, Johnson, Raye, \& Greene, 2004; Nyberg, Forkstam, Petersson, Cabeza, \& Ingvar, 2002).

Memory error and change blindness: overwriting and retrieval blocking

To detect a change, participants must consciously retrieve the identity of the object in the pre-change display at each location occupied by an object in the post-change display before they can make a comparison. Change blindness can arise from many sources, ranging from fragmented representation to comparison failure (see Simons, 2000 for a review). The overwriting hypothesis focuses on the nature of the pre-change representation (Beck \& Levin, 2003; Simons, 2000) by stating that the subsequent scene disrupts the pre-change representation so that only abstracted information from the initial scene remains. The pre-change representation is hence volatile and can support correct detection but not accurate recognition.

There is no doubt that overwriting leads to change blindness. Yet, the overwriting hypothesis cannot account for all the results (see Simons, 2000 for a review). Retrieval failure and comparison failure can also lead to change blindness (Hollingworth, 2003; Mitroff et al., 2004; ScottBrown et al., 2000; Simons et al., 2002). Although Mäntylä and Sundström (2004) demonstrated a recall bias, it is unclear whether the bias resulted from certain methodological characteristics and it is unclear what mechanisms underlie the recall bias. The results of this study suggest that the inadequate retrieval and recall bias may have arisen from retrieval blocking.

The retrieval-blocking hypothesis focuses on conscious retrieval of a pre-change representation rather than on the nature of its representation. The blocking hypothesis and the overwriting hypothesis are not mutually exclusive. The representation under change blindness can be as volatile as the overwriting hypothesis suggests (Beck \& Levin, 2003) or semi-complete (Simons \& Rensink, 2005 for commentary; Yeh \& Yang, 2008) in a given experimental context. In either context, the post-change object can interfere with access to the pre-change representation in conscious retrieval. The interference is greater when the pre-change object is not under focal attention during encoding than when the object is under focal attention. The interference is greater when both the pre- and post-change objects are encoded and compared under divided attention. A combination of overwriting and retrieval blocking can be detrimental to change detection.
When viewing a natural scene that contains multiple objects, participants must divide attention among various stimuli during encoding. The details of each object's properties may not be adequately acquired (Hollingworth, 2003) unless participants focus their attention on the object prior to comparison. As false recognition was as high as the hit rate when participants performed change detection under divided attention, memory error in viewing a natural scene may be inevitable. Note that the pre- and post-change objects in this study are unrelated. The frequency of false recognition may be even higher when the pre-change and post-change objects are related. As the perceived details of current stimuli can lead to false memory from reactivation and misattribution (Lyle \& Johnson, 2006), reactivation of pre-change features may be misattributed to the post-change object. The change in the presence of an object in a natural scene may also be difficult to detect because the thematic gist of a natural scene can lead to false recognition of exemplar objects that did not occur (Miller \& Gazzaniga, 1998).

Retrieval blocking certainly plays a role in change blindness by reducing the accessibility of the pre-change representation in conscious retrieval. Blocking can be released by providing a valid cue during encoding and comparison as shown in Experiment 5. Presenting a dot on the changed object in a video improves recognition under change blindness (Angelone, Levin, \& Simons, 2003). Presenting a cue before retrieval improves change detection (Hollingworth, 2003, 2005). When a useful retrieval cue was provided, participants could recall visual details of the pre-change object under change blindness (Simons et al., 2002). Knowledge about what can be changed in the visual world can also direct the retrieval of a pre-change object (Beck, Peterson, \& Angelone, 2007). Future research may uncover other methods that can release the blocking effect imposed by a post-change object upon the pre-change representation.

Acknowledgments This study was supported by grants (NSC952413-H-002-003, NSC96-2413-H-007-MY3) from the National Science Council. Parts of this work were presented in November 2005 at the 46th Annual Meeting of the Psychonomic Society, Toronto, Canada. We thank Y.-C. Tseng for her assistance on data collection. We thank anonymous reviewers for suggesting the addition of the "I don't remember" choice and the possibility of unequal attention to the pre- and post-change objects.

\section{References}

Agresti, A. (1996). An introduction to categorical data analysis. New York: Wiley.

Anderson, J. R. (1983). A spreading activation theory of memory. Journal of Verbal Learning and Verbal Behavior, 22, 261-295.

Anderson, M. C., \& Spellman, B. A. (1995). On the status of inhibitory mechanisms in cognition: Memory retrieval as a modal case. Psychological Review, 102, 68-100. 
Angelone, B. L., Levin, D. T., \& Simons, D. J. (2003). The roles of representation and comparison failures in change blindness. Perception, 32, 947-962.

Beck, M. R., \& Levin, D. T. (2003). The role of representational volatility in recognizing pre- and post-change objects. Perception \& Psychophysics, 65, 458-468.

Beck, M. R., Peterson, M. S., \& Angelone, B. L. (2007). The roles of encoding, retrieval, and awareness in change detection. Memory \& Cognition, 34, 610-620.

Bjork, R. A. (1989). Retrieval inhibition as an adaptive mechanism in human memory. In: H. L. Roediger, \& F. I. M. Craik (Eds.) Varieties of memory and consciousness: essays in Honour of Endel Tulving (pp. 309-330). Hillsdale: Lawrence Erlbaum Associates.

Brainerd, C. J., \& Reyna, V. F. (1996). Mere memory testing creates false memories in children. Developmental Psychology, 32, 467478.

Brainerd, C. J., \& Reyna, V. F. (1998). Fuzzy-trace theory and false memory: New frontiers. Journal of Experimental Child Psychology, 71, 194-209.

Brainerd, C. J., Wright, R., Reyna, V. F., \& Payne, D. G. (2002). Dual-retrieval processes in free and associative recall. Journal of Memory and Language, 46, 120-152.

CorelDRAW! [Computer Software]. (1994). Ottawa: Corel, Inc.

Hollingworth, A. (2003). Failures of retrieval and comparison constrain change detection in natural scenes. Journal of Experimental Psychology: Human Perception and Performance, 29, 388-403.

Hollingworth, A. (2005). The relationship between online visual representation of a scene and long-term scene memory. Journal of Experimental Psychology: Learning, Memory, and Cognition, 31, 396-411.

Hollingworth, A., Williams, C. C., \& Henderson, J. M. (2001). To see and remember: Visually specific information is retained in memory from previously attended objects in natural scenes. Psychonomic Bulletin \& Review, 8, 761-768.

Hopfinger, J. B., \& Mangun, G. R. (1998). Reflexive attention modulates processing of visual stimuli in human extrastriate cortex. Psychological Science, 9, 441-447.

Koch, K., Wagner, G., von Consbruch, K., Nenadic, I., Schultz, C., \& Ehle, C., et al. (2006). Temporal changes in neural activation during practice of information retrieval from short-term memory: an fMRI study. Brain Research, 1107, 140-150.

Libby, L. K., Neisser, U. (2001). Structure and strategy in the associative false memory paradigm. Memory, 9, 145-163.

Lyle, K. B., \& Johnson, M. K. (2006). Importing perceived features into false memories. Memory, 14, 197-213.

MacLeod, C. M. (1989). Directed forgetting affects both direct and indirect tests of memory. Journal of Experimental Psychology: Learning, Memory, and Cognition, 15, 13-21.

Mäntylä, T., \& Sundström, A. (2004). Changing scenes: Memory for naturalistic events following change blindness. Memory, 12, 696-706.

Miller, M. B., \& Gazzaniga, M. S. (1998). Creating false memories for visual scenes. Neuropsychologia, 36, 513-520.

Mitchell, K. J., Johnson, M. K., Raye, C. L., \& Greene, E. J. (2004). Prefrontal cortex activity associated with source monitoring in a working memory task. Journal of Cognitive Neuroscience, 16, 921-934.
Mitroff, S. R., Simons, D. J., \& Levin, D. T. (2004). Nothing compares 2 views: Change blindness results from failures to compare retained information. Perception \& Psychophysics, 66, $1268-1281$.

Noe, A., \& O'Regan, J. K. (2000). Perception, attention and the grand illusion. Psyche: an Interdisciplinary Journal of Research on Consciousness, 6.

Nyberg, L., Forkstam, C., Petersson, K. M., Cabeza, R., \& Ingvar, M. (2002). Brain imaging of human memory systems: Betweensystems similarities and within-system differences. Cognitive Brain Research, 13, 281-292.

Pé rez-Mata, M. N., Read, J. D., \& Diges, M. (2002). Effects of divided attention and word concreteness on correct recall and false memory reports. Memory, 10, 161-177.

Potter, M. C. (1976). Short-term conceptual memory for pictures. Journal of Experimental Psychology: Human Learning and Memory, 2, 509-522.

Raaijmakers, J. G. W., \& Shiffrin, R. M. (1981). Search of associative memory. Psychological Review, 88, 93-134.

Rensink, R. A. (2000). Seeing, sensing, and scrutinizing. Vision Research, 40, 1469-1487.

Roediger, H. L., \& McDermott, K. B. (1995). Creating false memories: Remembering words not presented in lists. Journal of Experimental Psychology: Learning, Memory, \& Cognition, 21, 803-814.

Roediger, H. L., Stellon, C. C., \& Tulving, E. (1977). Inhibition from part-list cues and rate of recall. Journal of Experimental Psychology: Human Learning and Memory, 2, 174-188.

Ryan, J. D., \& Cohen, N. J. (2004). The nature of change detection and online representations of scenes. Journal of Experimental Psychology: Human Perception and Performance, 30, 9881015.

Scott-Brown, K. C., Baker, M. R., \& Orbach, H. S. (2000). Comparison blindness. Visual Cognition, 7, 254-267.

Schacter, D. L. (2001). The seven sins of memory: How the mind forgets and remembers. New York: Houghton Mifflin Company.

Schmidt, B. K., Vogel, E. K., Woodman, G. F., \& Luck, S. J. (2002). Voluntary and automatic attentional control of visual working memory. Perception \& Psychophysics, 64, 754-763.

Schneider, W., Eschman, A., \& Zuccolotto, A. (2002). E-Prime user's guide. Pittsburgh: Psychology Software Tools Inc.

Silverman, M. E., \& Mack, A. (2006). Change blindness and priming: When it does and does not occur. Consciousness and Cognition, $15,409-422$.

Simons, D. J. (2000). Current approaches to change blindness. Visual Cognition, 7, 1-15.

Simons, D. J., \& Rensink, R. A. (2005). Change blindness: Past, present, and future. Trends in Cognitive Sciences, 9, 16-20.

Simons, D. J., Chabris, C. F., Schnur, T., \& Levin, D. T. (2002). Evidence for preserved representations in change blindness. Consciousness and Cognition, 11, 78-97.

Yantis, S., \& Jonides, J. (1984). Abrupt visual onsets and selective attention: evidence from visual search. Journal of Experimental Psychology: Human perception \& performance, 10, 601621.

Yeh, Y.-Y., \& Yang, C.-T. (2008). Object memory and change detection: Dissociation as a function of visual and conceptual similarity. Acta Psychologica, 127, 114-128. 